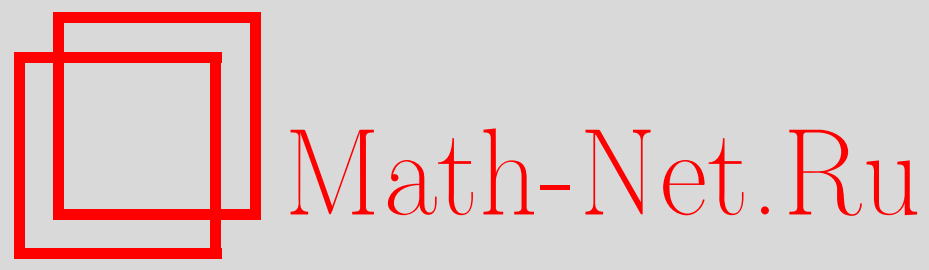

Г. Г. Ильюта, Интерполяция Лагранжа по системам Чебышёва и высшие порядки Брюа, Функи. анализ и его прил., 1998, том 32, выпуск 3, 76-79

DOI: https: //doi.org/10.4213/faa430

Использование Общероссийского математического портала MathNet.Ru подразумевает, что вы прочитали и согласны с пользовательским соглашением

http://www . mathnet.ru/rus/agreement

Параметры загрузки:

IP : 35.173 .219 .12

26 апреля 2023 г., 14:02:10

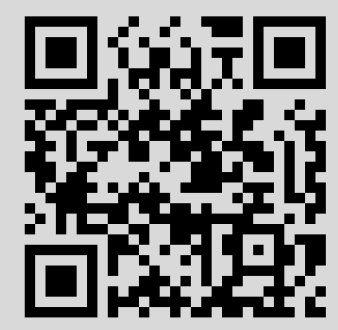




\title{
Интерполяция Лагранжа по системам Чебышёва и высшие порядки Брюа
}

\author{
(c) 1998. Г. Г. ИльюТА
}

С помощью интерполяции Лагранжа по системе функций $1, x, \ldots, x^{k-1}$ связной компоненте пространства конфигураций точек в $\mathbb{R}^{2}$ ставится в соответствие элемент высшего порядка Брюа [3, 4]. Конфигурация называется дискриминантной, если на графике некоторой линейной комбинации функций интерполирующего набора лежит больше, чем $k$, точек конфигурации. Рассмотрение в качестве конфигураций наборов точек экстремума полинома приводит к построению высшего исчисления змей В. И. Арнольда [1].

Ниже конструкция из $[3,4]$ обобшается в двух направлениях. Во-первых, рассматриваются конфигурации точек с кратностями. В этой ситуации высших порядков Брюа оказывается недостаточно, и мы определяем $\alpha$-высший порядок Брюа для каждого упорядоченного набора натуральных чисел $\alpha=$ $\left(n_{1}, \ldots, n_{q}\right)$ (высшие порядки Брюа из [8] отвечают наборам $\left.(1, \ldots, 1)\right)$. Это приводит к обобщению высших групп кос Манина-Шехтмана [8].

Другим обобщением является рассмотрение интерполяции по произвольному набору функций. Основной результат работы состоит в построении для интерполяции по системе Чебышёва (определение дано ниже) естественного отображения из множества связных компонент пространства общих конфигураций точек (с кратностями из набора $\alpha$ ) в $\alpha$-высшие порядки Брюа.

ОПРЕДЕЛЕНИЕ. Набор вещественных функций $f_{1}, \ldots, f_{k} \in C^{\infty}(D)$ на открытом интервале $D \subset \mathbb{R}$ называется системой Чебышёва, если любая ненулевая линейная комбинация функций этого набора имеет на $D$ меньше, чем $k$, корней (с учетом кратностей).

Определим кратные высшие порядки Брюа. Фиксируем $\alpha=\left(n_{1}, \ldots, n_{q}\right)$, $n=\sum n_{i}$. Рассмотрим множество, состоящее из $n_{i}$ чисел $i$ для $i=1, \ldots, q$, и обозначим через $C_{\alpha}(n, k)$ множество всех $k$-элементных подмножеств этого множества (равные элементы не различаются). Для $d \in C_{\alpha}(n, k+1)$ назовем пакетом в $C_{\alpha}(n, k)$ множество $\left\{l: l \in C_{\alpha}(n, k), l \subset d\right\}$. Число элементов в пакете равно числу различных элементов в $d$, и каждый элемент пакета получается из $d$ выбрасыванием некоторого элемента. Линейно упорядочим элементы пакета по величине выбрасываемого элемента и назовем этот порядок лексикографическим.

Линейный порядок на $C_{\alpha}(n, k)$ назовем допустимьлм, если он индуцирует на любом пакете либо лексикографический порядок, либо обратный ему. Обозначим через $A_{\alpha}(n, k)$ множество допустимых порядков на $C_{\alpha}(n, k)$. Два порядка из $A_{\alpha}(n, k)$ назовем элементарно эквивалентныли, если они отличаются только в паре соседних элементов, которые не принадлежат одному пакету.

ОПРЕДЕЛЕНИЕ. Назовем $\alpha$-выссиим порядком Брюа $B_{\alpha}(n, k)$ фактор $A_{\alpha}(n, k)$ по отношению эквивалентности, порожденному элементарной эквивалентностью. 
Частичный порядок на $B_{\alpha}(n, k)$ вводится аналогично тому, как это сделано в [8], с помощью обращения порядка на пакетах.

ПримеР. Пусть $\alpha=(2,1,3)$. Тогда

$$
\begin{aligned}
& C_{\alpha}(6,3)=\{112,113,233,133,123,333\}, \\
& C_{\alpha}(6,4)=\{1123,1133,1233,1333,2333\} .
\end{aligned}
$$

Выпишем отвечающие элементам $C_{\alpha}(6,4)$ пакеты в $C_{\alpha}(6,3)$ в лексикографическом порядке:

$$
\begin{aligned}
& 1123: 112,113,123 ; \\
& 1133: 113,133 ; \\
& 1233: 123,133,233 ; \\
& 1333: 133,333 ; \\
& 2333: 233,333 .
\end{aligned}
$$

Элементы множества $C_{\alpha}(6,3)$ выписаны выше в одном из допустимых порядков.

Определим $k$-общие наборы точек. Используем для этого классическую интерполяцию Лагранжа с кратными узлами [2]. Фиксируем $\alpha=\left(n_{1}, \ldots, n_{q}\right)$ и набор вещественных функций $f_{1}, \ldots, f_{k} \in C^{\infty}(D)$. Рассмотрим в $D \times \mathbb{R}$ множество конфигураций точек вида

$$
\begin{gathered}
\left(x_{1}, y_{1}^{1}\right), \ldots,\left(x_{1}, y_{1}^{n_{1}}\right),\left(x_{2}, y_{2}^{1}\right), \ldots,\left(x_{2}, y_{2}^{n_{2}}\right), \ldots,\left(x_{q}, y_{q}^{1}\right), \ldots,\left(x_{q}, y_{q}^{n_{q}}\right), \\
x_{1}<\cdots<x_{q} .
\end{gathered}
$$

Назовем подмножество конфигурации (1) начальнылм, если вместе с $\left(x_{i}, y_{i}^{p}\right)$ оно содержит все $\left(x_{i}, y_{i}^{j}\right), j<p$.

ОПРЕДЕлЕНИЕ. Конфигурацию (1) назовем $k$-общей (относительно $f_{1}, \ldots$, $\left.f_{k}\right)$, если ни для какого $(k+1)$-элементного начального подмножества $S$ не существует линейной комбинации $\sum a_{j} f_{j}$, обладающей свойством

$$
y_{i}^{p}=\sum a_{j} f_{j}^{(p-1)}\left(x_{i}\right) \quad \text { для всех }\left(x_{i}, y_{i}^{p}\right) \in S .
$$

СЛЕДСТВИЕ ОПРЕДЕЛЕНИЯ. Пусть $f_{1}, \ldots, f_{k} \in C^{\infty}(D)$ - cucmeмa Чeбышёва. Тогда для любого $k$-элементного начального подмножества $S$ k-общей конфигурачии (1) существует единственная линейная комбиначия $\sum a_{j} f_{j}$ (назовем ее полиномом конфигурации (1)), удовлетворлющал условию (2) для всех точек из $S$.

Сопоставим $k$-общей конфигурации $F$, определенной по системе Чебышёва, множество линейных порядков на $C_{\alpha}(n, k)$. Для любого $x_{0}>x_{q}, x_{0} \in D$, рассмотрим точки пересечения прямой $x=x_{0}$ с графиками всех полиномов конфигурации. Линейно упорядочим точки пересечения по величине координаты $y$ (совпадающие упорядочиваем произвольно). Это дает линейный порядок на $C_{\alpha}(n, k)$. Обозначим через $c(F)$ множество всех порядков, которые так можно получить. 
Теорема 1. Пусть $F$ есть $k$-общая конфигурачия точек, определенная по системе Чебышёва. Тогда все линейнье порядки в $c(F)$ допустимь и эквивалентны, т.е. $F$ определяет әлемент из $B_{\alpha}(n, k)$. Построенное отображение постоянно на компонентах свлзности пространства $k$-общих конфигурачий.

ДокаЗАТЕльство. Теорему 1 можно получить как обобщение соответствующего факта из [4], но мы приведем другое доказательство. Достаточно доказать, что 1) все порядки из $c(F)$ индуцируют на каждом пакете один и тот же порядок, 2) этот порядок является лексикографическим или обратным к нему порядком. Выберем пакет и обозначим через $P_{1}, P_{2}, \ldots$ отвечающие этому пакету полиномы конфигурации в лексикографическом порядке. Для всех $i, j$ полином $P_{i}-P_{j}$ имеет на $D$ меньше $k$ корней. Поэтому точками пересечения (с кратностями) графиков полиномов $P_{i}$ являются только точки конфигурации, отвечающие выбранному пакету. Отсюда сразу вытекает 1), поскольку для $x_{0}>x_{q}$ прямая $x=x_{0}$ не содержит точек пересечения графиков полиномов $P_{i}$ и поэтому порядок точек пересечения этой прямой с графиками не зависит от $x_{0}$. Ключевое замечание доказательства выделим как лемму.

Лемма. Топология конфигурации графиков полиномов $P_{i}$ не зависит от системь Чебышёва.

Действительно, из непрерывности функций $P_{i}$ вытекает, что топология конфигурации графиков зависит только от пересечений этих графиков, но, согласно сказанному выше, пересечения определяются пакетом и не зависят от системы Чебышёва.

Таким образом, можно считать, что интерполирующей системой является система $1, x, \ldots, x^{k-1}$ и $P_{i}$ - обычные полиномы степени не выше $k-1$. Порядок на точках пересечения графиков полиномов $P_{i}$ с прямой $x=x_{0}>x_{q}$ совпадает с порядком на коэффициентах при $x^{k-1}$ полиномов $P_{i}$. Коэффициент при $x^{k-1}$ в интерполяционном полиноме Лагранжа - это разделенная разность, построенная по интерполируемому набору точек [2]. Рекуррентная формула для разделенных разностей [2] означает, что разность коэффициентов при $x^{k-1}$ для двух полиномов $P_{i}, P_{j}$ равна разделенной разности для отвечающих всему пакету точек конфигурации, умноженной на $x_{i}-x_{j}$. Поэтому для всех $i<j$ разности коэффициентов при $x^{k-1}$ полиномов $P_{i}, P_{j}$ имеют один и тот же знак. Теорема доказана.

ЗАмЕчАния 1. Если рассматривать интерполяцию по набору функций любого числа переменных, то естественным комбинаторным объектом, который можно сопоставить конфигурации точек, является ориентированный матроид [5]. Отвечающие элементам высших порядков Брюа ориентированные матроиды характеризуются в $[7,9]$.

2. Если фиксировать координаты $x$ в конфигурациях точек, то дискриминант в пространстве конфигураций будет конфигурацией гиперплоскостей в $n$-мерном пространстве координат $y$ [4]. Над $\mathbb{C}$ фундаментальная группа дополнения к этой конфигурации гиперплоскостей обобщает высшие группы кос [8] для кратных высших порядков Брюа $B_{\alpha}(n, k)$. 
3. Теорема 1 может быть обобщена для интерполяции по симметрическим функциям [6]. Мы займемся этим в другой статье.

4. Условия гладкости на интерполирующий набор функций в теореме 1 могут быть ослаблены, но для ясности мы жертвуем несущественной общностью.

\title{
ЛИТЕРАТУРА
}

1. Арнольд В. И. УМН, 47, вып. 1, 3-45 (1992). 2. Гельфонд А. О. Исчисление конечных разностей. Наука, М., 1967. 3. Ильюта Г. Г. Доклады РАН, 347, № 3, 217-219 (1996). 4. Ильюта Г. Г. Изв. РАН, сер. матем., 60, вып. 6, 91-100 (1996). 5. Bjorner A., Las Vergnas M., Sturmfels B., White N., Ziegler G. Oriented matroids. Cambridge Univ. Press, Cambridge, 1993. 6. Chen W., Louck J. Adv. Math., 117, 147-156 (1996). 7. Kapranov M., Voevodsky V. Combinatorial geometric aspects of polycategory theory: pasting schemes and higher Bruhat orders. Cahiers de Topol. et Geom., 1991, v. XXXII-1, pp. 11-27. 8. Manin Yu., Schechtman V. Adv. Stud. Pure Math., 17, 289-308 (1989). 9. Ziegler G. Topology, 32, No. 2, 259-279 (1993).

Государственная академия

Поступило в редакцию нефти и газа им. И. М. Губкина

2 апреля 1997 г.

УДК 517.9

\section{Существование и единственность сохраняющего меру оптимального отображения в общей задаче Монжа-Канторовича}

\author{
(c) 1998. В. Л. ЛЕвин
}

1. Введение. Пусть $X, Y$ - польские пространства, $\sigma_{1}$ и $\sigma_{2}$ - конечные положительные борелевские меры на них с $\sigma_{1} X=\sigma_{2} Y$ и $c$ - непрерывная функция на $X \times Y$. Далее $\pi_{1}$ и $\pi_{2}$ обозначают естественные проектирования пространства $X \times Y$ на $X$ и $Y$. Под общей задачей Монжа-Канторовича мы понимаем экстремальную задачу, состоящую в нахождении оптимального значения

$$
\mathscr{C}\left(c ; \sigma_{1}, \sigma_{2}\right):=\inf \left\{c(\mu): \mu \in \Gamma\left(\sigma_{1}, \sigma_{2}\right)\right\},
$$

где $c(\mu)$ - интеграл функции $c$ по $\mu$ (считаем, что по определению $c(\mu)=$ $+\infty$, если $\left.c_{+}(\mu)=+\infty\right)$, а $\Gamma\left(\sigma_{1}, \sigma_{2}\right)$ - множество положительных борелевских мер $\mu$ на $X \times Y$, для которых $\sigma_{1}$ и $\sigma_{2}$ являются их маргинальными мерами: $\pi_{1}(\mu)=\sigma_{1}, \pi_{2}(\mu)=\sigma_{2}$. Положим

$$
\mathscr{V}\left(c ; \sigma_{1}, \sigma_{2}\right):=\inf \left\{\int_{X} c(x, f(x)) \sigma_{1}(d x): f \in \Phi\left(\sigma_{1}, \sigma_{2}\right)\right\},
$$

где $\Phi\left(\sigma_{1}, \sigma_{2}\right)$ — множество сохраняющих меру отображений $f: X \rightarrow Y$. Борелевское отображение $f$ называется сохраняющим меру, если $f\left(\sigma_{1}\right)=\sigma_{2}$,

^ Работа выполнена при поддержке РФФИ, грант 96-01-00336. 